\title{
On Decoupling of Functions of Normal Vectors II
}

\author{
Pavel G. Grigoriev and Stanislav A. Molchanov \\ Department of Mathematics and Statistics \\ University of North Carolina at Charlotte \\ Charlotte, NC 28223, USA \\ thepavel@mail.ru
}

\begin{abstract}
A decoupling type inequality for a sum of functions of Guassian vectors is established.

Key words: Decoupling, Gaussian vectors, Wick's polynomials, Hermite's polynomials.
\end{abstract}

\section{Results}

In [3] the following decoupling results were established.

Theorem 1.1. For a normally distributed random vector $\bar{Y}=\left(Y_{i}\right)_{i=1, \ldots, d}$ satisfying $\mathrm{E} Y_{i}=0, \mathrm{E} Y_{i}^{2}=1, i=1, \ldots, d$, we have

$$
c^{-} \sum_{i=1}^{d}\left\|\varphi_{i}\left(Y_{i}\right)\right\|_{2}^{2} \leq\left\|\sum_{i=1}^{d} \varphi_{i}\left(Y_{i}\right)\right\|_{2}^{2} \leq c^{+} \sum_{i=1}^{d}\left\|\varphi_{i}\left(Y_{i}\right)\right\|_{2}^{2}
$$

for all measurable functions $\varphi_{i}: \mathbb{R} \rightarrow \mathbb{R}$ satisfying $\mathrm{E} \varphi_{i}\left(Y_{i}\right)=0$ with the constants $c^{-}$and $c^{+}$being the smallest and the largest eigenvalues of the correlation matrix of $\bar{Y}$. Moreover the constants are the best possible.

Theorem 1.2. Let $\bar{Y}_{1}=\left(Y_{1, i}\right)_{i=1, \ldots, d_{1}}$ and $\bar{Y}_{2}=\left(Y_{2, j}\right)_{j=1, \ldots, d_{2}}$ be standard normal vectors with the correlations

$$
\mathrm{E} Y_{\alpha, i} Y_{\beta, j}= \begin{cases}0, & \alpha=\beta, i \neq j \\ 1, & \alpha=\beta, i=j \\ \rho_{i, j}, & \alpha \neq \beta\end{cases}
$$

Then

$$
c_{-}\left(\left\|\varphi_{1}\left(\bar{Y}_{1}\right)\right\|_{2}^{2}+\left\|\varphi_{2}\left(\bar{Y}_{2}\right)\right\|_{2}^{2}\right) \leq\left\|\varphi_{1}\left(\bar{Y}_{1}\right)+\varphi_{2}\left(\bar{Y}_{2}\right)\right\|_{2}^{2} \leq c_{+}\left(\left\|\varphi_{1}\left(\bar{Y}_{1}\right)\right\|_{2}^{2}+\left\|\varphi_{2}\left(\bar{Y}_{2}\right)\right\|_{2}^{2}\right)
$$

holds for all measurable functions $\varphi_{\alpha}: \mathbb{R}^{d_{\alpha}} \rightarrow \mathbb{R}$ such that $\mathrm{E} \varphi_{\alpha}\left(\bar{Y}_{\alpha}\right)=0, \alpha=1,2$, with the constants $c_{ \pm}=1 \pm s^{*}$, where $s^{*}$ is the maximum singular value of the matrix $R=\left(\rho_{i, j}\right)_{i=1, \ldots, d_{1}, j=1, \ldots, d_{2}}$. These constants cannot be improved. 
These theorems refine the estimates used by Cherny et al. [1], [2]. Here we generalize these results further and prove

Theorem 1.3. Let $\bar{Y}_{\alpha}=\left(Y_{\alpha, i}\right)_{i=1, \ldots, d_{\alpha}}, \alpha=1, \ldots, N$, be standard normal vectors with the correlations

$$
\mathrm{E} Y_{\alpha, i} Y_{\beta, j}= \begin{cases}0, & \alpha=\beta, i \neq j \\ 1, & \alpha=\beta, i=j \\ \rho_{i, j}^{\alpha, \beta}, & \alpha \neq \beta\end{cases}
$$

Then

$$
C_{-} \sum_{\alpha=1}^{N}\left\|\varphi_{\alpha}\left(\bar{Y}_{\alpha}\right)\right\|_{2}^{2} \leq\left\|\sum_{\alpha=1}^{N} \varphi_{\alpha}\left(\bar{Y}_{\alpha}\right)\right\|_{2}^{2} \leq C_{+} \sum_{\alpha=1}^{N}\left\|\varphi_{\alpha}\left(\bar{Y}_{\alpha}\right)\right\|_{2}^{2}
$$

for all measurable functions $\varphi_{\alpha}: \mathbb{R}^{d_{\alpha}} \rightarrow \mathbb{R}$, satisfying $\mathrm{E} \varphi_{\alpha}\left(\bar{Y}_{\alpha}\right)=0$ for all $\alpha=1, \ldots, N$. The constants $C_{ \pm}=1 \pm \sigma_{0}$, where $\sigma_{0}$ denotes the largest eigenvalue of the matrix $S^{*}=\left(s_{\alpha, \beta}^{*}\right)_{1 \leq \alpha, \beta \leq N}$ with $s_{\alpha, \beta}^{*}$ being the maximum singular values of the matrices $R^{\alpha, \beta}=\left(\rho_{i, j}^{\alpha, \beta}\right)_{i=1, \ldots, d_{\alpha}, j=1, \ldots, d_{\beta}}$ for $\alpha \neq \beta$ and $s_{\alpha, \alpha}^{*}:=0$.

Note that while Theorems 1.1 and 1.2 give the decoupling estimates with sharp constants, Theorem 1.3 provides just rough estimate with not the best constants (in particular $C_{-}$could be negative). However, in view of the applications described in Cherny et al. [1], 2] the upper bound in (11) is still interesting.

\section{Proof of Theorem 1.3}

The proof of Theorem 1.3 follows the framework used in [3]. We need to introduce notations used in [3] and borrowed from [4].

For $k=0,1, \ldots$ we define Wick's polynomials $: x^{k}:$ by the extension

$$
\exp \left(a x-\frac{a}{2}\right)=\sum_{k=0}^{\infty} a^{k} \frac{x^{k}:}{k !} .
$$

(Wick's polynomials are specially normalized Hermite's polynomials used in mathematical physics. We find these notations convenient for multidimensional case.)

Let $\bar{k}=\left(k_{1}, \ldots, k_{d}\right)$ be a $d$-dimensional vector of non-negative integers. Set

$$
\begin{aligned}
|\bar{k}| & :=k_{1}+\cdots+k_{d}, \\
\bar{k} ! & :=k_{1} ! k_{2} ! \ldots k_{d} ! \\
\bar{a}^{\bar{k}} & :=a_{1}^{k_{1}} a_{2}^{k_{2}} \ldots a_{d}^{k_{d}}, \quad \text { for } \bar{a}:=\left(a_{i}\right)_{i=1, \ldots, d} \in \mathbb{R}^{d} .
\end{aligned}
$$

For a vector variable $\bar{x}=\left(x_{k}\right)_{k=1}^{d}$ we define multidimensional Wick's polynomial by

$$
: \bar{x}^{\bar{k}}::=\prod_{i=1}^{d}: x_{i}^{k_{i}}:
$$


It is well known (see e.g. 44) that for a standard $d$-dimensional normal vector $\bar{Y}$ the system $\left\{\bar{k} !^{-1 / 2}: \bar{Y}^{\bar{k}}:\right\}_{\bar{k} \in \mathbb{Z}_{0}^{d}}$ is an orthonormal bases in the $L_{2}$ space generated by all square-integrable $f(\bar{Y})$. So for each $\alpha=1, \ldots, N$, we have

$$
\varphi_{\alpha}\left(\bar{Y}_{\alpha}\right)=\sum_{\bar{k} \in \mathbb{Z}_{0}^{d_{\alpha}}} a_{\alpha, \bar{k}} \frac{: \bar{Y}_{\alpha}^{\bar{k}}:}{\bar{k} !^{1 / 2}}=\sum_{n=0}^{\infty} \sum_{\bar{k} \in \mathbb{Z}_{0}^{d_{\alpha}},|\bar{k}|=n} a_{\alpha, \bar{k}} \frac{: \bar{Y}_{\alpha}^{\bar{k}}:}{\bar{k} !^{1 / 2}}
$$

and therefore

$$
\sum_{\alpha=1}^{N} \varphi_{\alpha}\left(\bar{Y}_{\alpha}\right)=\sum_{\alpha=1}^{N} \sum_{n=0}^{\infty} \sum_{\bar{k}_{\alpha} \in \mathbb{Z}_{0}^{d_{\alpha}},\left|\bar{k}_{\alpha}\right|=n} a_{\alpha, \bar{k}_{\alpha}} \frac{: \bar{Y}_{\alpha}^{\bar{k}_{\alpha}}:}{\bar{k}_{\alpha} !^{1 / 2}}=\sum_{n=0}^{\infty} \sum_{\alpha=1}^{N} P_{n}\left(\bar{Y}_{\alpha}\right)
$$

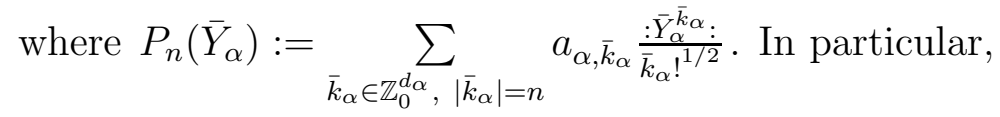

$$
\sum_{\alpha=1}^{N}\left\|\varphi_{\alpha}\left(\bar{Y}_{\alpha}\right)\right\|_{2}^{2}=\sum_{n=0}^{\infty} \sum_{\alpha=1}^{N}\left\|P_{n}\left(\bar{Y}_{\alpha}\right)\right\|_{2}^{2}
$$

It is well-known that $P_{n_{1}}\left(\bar{Y}_{\alpha}\right)$ and $P_{n_{2}}\left(\bar{Y}_{\beta}\right)$ are orthogonal whenever $n_{1} \neq n_{2}$ (see e.g. [3]). Consequently, to prove (1) for arbitrary $\varphi_{\alpha}$ it suffices to prove it for $\phi_{\alpha}\left(\bar{Y}_{\alpha}\right)=P_{n}\left(\bar{Y}_{\alpha}\right)$ for each $n=0,1, \ldots$.

For a fixed $n$, we have

$$
\left\|\sum_{\alpha=1}^{N} P_{n}\left(\bar{Y}_{\alpha}\right)\right\|_{2}^{2}=\sum_{\alpha=1}^{N}\left\|P_{n}\left(\bar{Y}_{\alpha}\right)\right\|_{2}^{2}+2 \sum_{\alpha<\beta} \mathrm{E}\left[P_{n}\left(\bar{Y}_{\alpha}\right) P_{n}\left(\bar{Y}_{\beta}\right)\right] .
$$

Fix a pair $\alpha<\beta$. Let $R^{\alpha, \beta}=U \Sigma V^{T}$ be the singular value decomposition of the matrix $R^{\alpha, \beta}$ (recall that here $\Sigma$ is a $d_{\alpha} \times d_{\beta}$ diagonal matrix whose diagonal entries are the singular values of $R^{\alpha, \beta}$ and $U, V$ are orthogonal matrices of corresponding sizes). Let $\bar{Z}_{1}:=U \bar{Y}_{\alpha}$ and $\bar{Z}_{2}:=V \bar{Y}_{\beta}$ (all the vectors are assumed being columns). This transformation can be written in the block matrix form

$$
\left(\begin{array}{c}
\bar{Z}_{1} \\
\bar{Z}_{2}
\end{array}\right)=\left(\begin{array}{cc}
U & 0 \\
0 & V
\end{array}\right)\left(\begin{array}{c}
\bar{Y}_{\alpha} \\
\bar{Y}_{\beta}
\end{array}\right)
$$

with the obviously orthogonal $\left(d_{\alpha}+d_{\beta}\right) \times\left(d_{\alpha}+d_{\beta}\right)$ transformation matrix. It follows that

$$
\mathrm{E}\left[P_{n}\left(\bar{Y}_{\alpha}\right) P_{n}\left(\bar{Y}_{\beta}\right)\right]=\mathrm{E}\left[P_{n}\left(\bar{Z}_{1}\right) P_{n}\left(\bar{Z}_{2}\right)\right]=\sum_{\substack{\bar{k}_{\alpha} \in \mathbb{Z}_{\alpha}^{d_{\alpha}} \\\left|\bar{k}_{\alpha}\right|=n \\ \bar{k}_{\beta} \in \mathbb{Z}_{0}^{d_{\beta}} \\\left|\bar{k}_{\beta}\right|=n}} a_{\alpha, \bar{k}_{\alpha}} a_{\beta, \bar{k}_{\beta}} \mathrm{E} \frac{: \bar{Z}_{1}^{\bar{k}_{\alpha}}:: \frac{\bar{Z}_{2}^{\bar{k}_{\beta}}}{\bar{k}_{\alpha} !^{1 / 2}}}{\bar{k}_{\beta} !^{1 / 2}}
$$

Note that the covariance structure of $\left(\bar{Z}_{1}, \bar{Z}_{2}\right)$ is relatively simple, its covariance matrix is

$$
\left(\begin{array}{cc}
I_{d_{\alpha}} & \Sigma \\
\Sigma^{T} & I_{d_{\beta}}
\end{array}\right)
$$


Without loss of generality we assume that $d_{\alpha} \geq d_{\beta}$, i.e. the vectors $\bar{Y}_{\alpha}$ are ordered according to their dimensions. Let us agree that the index vectors of different dimensions are equal $\left(\bar{k}_{\alpha}=\bar{k}_{\beta}\right)$ if the shorter vector $\bar{k}_{\beta}$ coincides with the first $d_{\beta}$ entries of $k_{\alpha}$ and the other entries of $k_{\alpha}$ are zeros. Also let us denote by $\bar{s}$ the vector of the diagonal entries of $\Sigma$ (i.e. the singular values of $R^{\alpha, \beta}$ ).

Using Lemma 3.1 from [3] (for the case of diagonal $R$, see also formula (3.5) in [3]) we conclude

$$
\mathrm{E}: \bar{Z}_{1}^{\bar{k}_{\alpha}}:: \bar{Z}_{2}^{\bar{k}_{\beta}}:= \begin{cases}0, & \bar{k}_{\alpha} \neq \bar{k}_{\beta} \\ \bar{k}_{\beta} ! \bar{s}^{\bar{k}_{\beta}}, & \bar{k}_{\alpha}=\bar{k}_{\beta}\end{cases}
$$

Using (4) we continue (3) as follows

$$
\mathrm{E}\left[P_{n}\left(\bar{Y}_{\alpha}\right) P_{n}\left(\bar{Y}_{\beta}\right)\right]=\sum_{\substack{d_{\beta} \\ \bar{k}_{\beta} \in \mathbb{Z}_{0}, \bar{k}_{\alpha} \in \mathbb{Z}_{\alpha}^{d_{\alpha}} \\\left|\bar{k}_{\beta}\right|=n, k_{\alpha}=\bar{k}_{\beta}}} a_{\alpha, \bar{k}_{\alpha}} a_{\beta, \bar{k}_{\beta}} \bar{s}^{\bar{s}_{\beta}} .
$$

Taking into account that the singular values lie in $[0,1]$ (because they are certain correlations) we can estimate

$$
\begin{aligned}
& \left|\mathrm{E}\left[P_{n}\left(\bar{Y}_{\alpha}\right) P_{n}\left(\bar{Y}_{\beta}\right)\right]\right| \leq s_{\alpha, \beta}^{*} \sum_{\substack{d_{\beta} \\
\bar{k}_{\beta} \in \mathbb{Z}_{0}, \bar{k}_{\alpha} \in \mathbb{Z}_{0}^{d_{\alpha}} \\
\left|\bar{k}_{\beta}\right|=n, k_{\alpha}=\bar{k}_{\beta}}}\left|a_{\alpha, \bar{k}_{\alpha}} a_{\beta, \bar{k}_{\beta} \mid}\right| \\
& \leq s_{\alpha, \beta}^{*}\left(\sum_{\substack{\bar{k}_{\alpha} \in \mathbb{Z}_{\alpha}^{d_{\alpha}} \\
\left|\bar{k}_{\alpha}\right|=n}} a_{\alpha, \bar{k}_{\alpha}}^{2}\right)^{\frac{1}{2}}\left(\sum_{\substack{\bar{k}_{\beta} \in \mathbb{Z}_{0}^{d_{\beta}} \\
\left|\bar{k}_{\beta}\right|=n}} a_{\beta, \bar{k}_{\beta}}^{2}\right)^{\frac{1}{2}}=s_{\alpha, \beta}^{*}\left\|P_{n}\left(\bar{Y}_{\alpha}\right)\right\|_{2}\left\|P_{n}\left(\bar{Y}_{\beta}\right)\right\|_{2}
\end{aligned}
$$

Recalling (2) we conclude

$$
\left|\left\|\sum_{\alpha=1}^{N} P_{n}\left(\bar{Y}_{\alpha}\right)\right\|_{2}^{2}-\sum_{\alpha=1}^{N}\left\|P_{n}\left(\bar{Y}_{\alpha}\right)\right\|_{2}^{2}\right| \leq 2 \sum_{\alpha<\beta} s_{\alpha, \beta}^{*}\left\|P_{n}\left(\bar{Y}_{\alpha}\right)\right\|_{2}\left\|P_{n}\left(\bar{Y}_{\beta}\right)\right\|_{2} .
$$

By standard linear algebra argument we have that the right hand side is bounded by $\sigma_{0} \sum_{\alpha=1}^{N}\left\|P_{n}\left(\bar{Y}_{\alpha}\right)\right\|_{2}^{2}$, where $\sigma_{0}$ is the largest eigenvalue of the matrix $S^{*}$. We conclude that for a fixed $n$ the estimate (11) holds for $\phi_{\alpha}\left(\bar{Y}_{\alpha}\right)=P_{n}\left(\bar{Y}_{\alpha}\right), \alpha=1, \ldots, N$, with $C_{ \pm}=1 \pm \sigma_{0}$. As it was already pointed out this means that we have (1) for all $\phi_{\alpha}$ with the same constants.

\section{References}

[1] Cherny, A., Douady, R., Molchanov, S. On measuring nonlinear risk with scarce observations. Finance Stoch. 14 (2010), no. 3, p. 375-395.

[2] Cherny, A., Douady, R., Molchanov, S. On Measuring Hedge Fund Risk. 2008. Preprint, available at: http://mech.math.msu.su/ ${ }^{\sim}$ cherny.

[3] Grigoriev, P., Molchanov, S. On Decoupling of Functions of Normal Vectors. Math. Notes 92 (2012), p. 401-409.

[4] Malyshev, V.A., Minlos, R.A. Gibbs Random Fields. Kluwer, Dordrecht (1991) 